\title{
Aprender, servir y ser solidarios en tiempos de pandemias
}

\author{
María Rosa Tapia \\ Andrés Peregalli
}

Centro Latinoamericano de Aprendizaje y Servicio Solidario (CLAYSS)

\section{Resumen}

En tiempos de pandemias se desafía la capacidad de individuos, colectivos sociales, organizaciones, instituciones, políticas y gobiernos, para crear y recrear el aprendizaje, el servicio y la solidaridad. Sustentado en el marco del Aprendizaje y Servicio Solidario (AYSS) y una perspectiva organizacional estratégica, se analiza el mapa "Aprendizaje-servicio en tiempos de pandemia" (Centro Latinoamericano de Aprendizaje y Servicio Solidario [CLAYSS], 2020). Se identifican, exploran y analizan 200 experiencias de AYSS, georreferenciadas en cuatro continentes y 22 países entre marzo y septiembre de 2020. Se utiliza la técnica de análisis de contenido temático y la perspectiva etnográfica digital para sistematizar y categorizar las experiencias. El análisis evidencia el devenir del AYSS en tiempos de pandemias, sus énfasis, acciones y búsquedas, y muestra la permanencia del foco de los proyectos en el protagonismo de quienes aprenden, el vínculo con la comunidad y la integración del servicio solidario en el curriculum. El mapa denota un habitus organizacional (aprender de y con otros); su diseño y desarrollo sustentan la perspectiva de una construcción colectiva, de un conocimiento distribuido que recrea esperanza e invita a (re)construir redes solidarias en tiempos de pandemias.

\section{Palabras clave}

Aprendizaje-servicio solidario, pandemia, etnografía digital, aprendizaje organizacional. 


\title{
Learning, serving and being solidary in times of a pandemic
}

\begin{abstract}
In times of a pandemic, the ability of individuals, social groups, organizations, institutions, policies and governments to create and recreate learning, service and solidarity is severely challenged. Based on the framework of solidarity service-learning and a strategic organizational perspective, this paper analyzes the 'Service-Learning in times of a pandemic' map. Two hundred solidarity service-learning experiences were identified, explored and analysed, georeferenced in four continents and 22 countries, between March and September 2020. The thematic content analysis technique and the digital ethnographic perspective were used to systematize and categorize the experiences. The analysis shows the evolution of solidarity service-learning in times of a pandemic, its focal point, actions and goals, evidencing how the projects have remained focused on the learners' central role, the bond with the community and the integration of solidarity service into the curriculum. The map denotes an organizational habitus (learning from and with others); its design and development supports the perspective of a collective construction of knowledge, which is shared and generates recreates hope and invites us to (re) build solidarity networks in times of pandemics. The map is evidence of the interweaving of solidarity service-learning citizenship (political action), rights and education, which systematizes and enhances a way of being-making-knowing that has been shared for decades between local and global actors and the networks that are configured in times of a pandemic.
\end{abstract}

\section{Keywords}

Solidarity service-learning, pandemic, digital ethnography, organizational learning. 


\section{Introducción}

A mediados de abril de 2020, casi todos los países del planeta habían cerrado sus escuelas, y otras instituciones educativas, en respuesta a la pandemia de COVID-19, que afectó a más de 1570 millones de alumnos $(90 \%$ del total de la población escolar matriculada en el mundo).

Actualmente, y tal como lo muestra el mapa "Seguimiento Mundial de los cierres de escuelas causados por el COVID-19" (Organización de las Naciones Unidas para la Educación, la Ciencia y la Cultura [UNESCO], 2020) más de 850 millones de estudiantes experimentan el cierre de dichas instituciones en el mundo ( $50 \%$ del total de alumnos matriculados en el mundo; 53 países. UNESCO, 2020). En este contexto se implementaron diversas iniciativas orientadas a sostener la escolaridad en un contexto en que se profundizaron las desigualdades. Diversas propuestas surgieron para continuar los procesos de enseñanza a través de los medios disponibles (o viables/factibles) en cada contexto: plataformas digitales con recursos para docentes, estudiantes y familias; televisión y radio, con clases y programas educativos de entretenimiento; materiales impresos destinados a estudiantes sin conectividad. Las alternativas fueron similares, pero no los tiempos de implementación, dada la disparidad de plataformas públicas educativas y de comunicación disponibles (Ministerio de Educación, 2020).
La pandemia del COVID-19, que se suma a otras pandemias que experimenta el mundo, desafía nuestra capacidad de recrear solidaridad y esperanza, servicio y aprendizaje. Tal como expresó el Papa Francisco (2020) "Si actuamos como un solo pueblo, incluso ante las otras epidemias que nos acechan, podemos lograr un impacto real" (p.50); "una emergencia como la del COVID-19 es derrotada en primer lugar con los anticuerpos de la solidaridad" (Pontificia Academia para la Vida, 2020, párr.13). En este marco los recursos digitales se han convertido en potentes herramientas capaces de conectar personas y colectivos sociales que habitan la aldea global, formidables recursos que pueden nutrir ( $y$ potenciar) el ciclo de aprendizaje de las organizaciones y redes de aprendizaje y servicio solidario (AYSS) en el mundo, fortaleciendo lazos preexistentes y multiplicándose.

Este artículo presenta el mapa "Aprendizaje-servicio en tiempos de pandemia" (CLAYSS, 2020), como evidencia de un tejido de AYSS, ciudadanía y educación que recoge y potencia un trabajo compartido desde hace décadas entre diferentes actores y redes del mundo y se potencia en estos tiempos. Se ofrece como evidencia de un habitus organizacional (aprender de y con otros) que se nutre de experiencias y contribuye a nutrirlas. Más que la imagen estática de un mapa (o el mapa exclusivamente como recurso), su diseño y desarrollo refiere a una construcción colectiva, a una distribución del conocimiento que recrea esperanza y construye redes solidarias en tiempos de pandemias. 


\section{Marco teórico}

Este artículo se basa en cuatro conceptos clave: AYSS, aprendizajeservicio virtual o e-service-learning, etnografía digital y aprendizaje organizacional. Estos conceptos sustentan el análisis de la experiencia referenciada (Algunas experiencias de aprendizaje-servicio solidario frente a epidemias, CLAYSS, 2020) a efectos de contribuir al desarrollo de conocimiento y conceptualización en estos tiempos, así como con la esperanza de motivar a docentes e instituciones educativas alrededor del mundo a iniciar $\mathrm{y} / \mathrm{o}$ sostener prácticas socioeducativas solidarias aún en este contexto.

El Aprendizaje y Servicio Solidario (en adelante AYSS) es un movimiento pedagógico innovador que promueve actividades estudiantiles solidarias en que los conocimientos se aplican a la resolución de problemáticas y necesidades concretas de la comunidad. Los proyectos de AYSS contribuyen simultáneamente al desarrollo local y a mejorar la calidad del aprendizaje académico, al desarrollo de competencias adecuadas para la inserción en el mundo del trabajo, la formación personal en valores, y la participación ciudadana responsable. En función de los consensos más generalizados, se entienden como prácticas de AYSS aquellas que reúnen simultáneamente al menos tres características (Tapia, 2000):

- servicio solidario destinado para atender en forma acotada y eficaz necesidades reales y sentidas con una comunidad, y no sólo para ella,
- protagonizado activamente por los estudiantes desde el planeamiento a la evaluación,

- articulado intencionadamente con los contenidos de aprendizaje, es decir involucrando los contenidos curriculares, reflexión sobre la práctica, desarrollo de competencias para la ciudadanía y el trabajo, e investigación vinculada a la práctica solidaria (pp. 26-27).

Este momento de la humanidad nos desafía (e interpela) a recrear el aprendizaje, el servicio y la solidaridad, durante las pandemias y proyectar el después; y conceptualizar las prácticas y experiencias que se realizan en diferentes planos, ámbitos y modalidades. El análisis y la reflexión acerca de este tiempo y los proyectos implementados permiten definir diferentes ámbitos y modalidades de acción del AYSS. Tomando como referencia la categorización del aprendizaje-servicio virtual o e-servicelearning realizada por Waldner, McGorry y Widener (2012), en tiempos de aislamiento social donde las actividades de aprendizaje se trasladaron en gran medida a la virtualidad, podemos identificar tres categorías (CLAYSS Digital, 2020, 1h27m35s):

- AYSS presencial: Ios proyectos y actividades se realizan en la comunidad o en aulas y talleres, manteniendo "distancia social y los correspondientes cuidados". La solidaridad presencial sigue siendo indispensable porque las desigualdades se han agravado; "coraje" y "cuidado" y "ética del 
riesgo" (Piro, 2020) son las

palabras clave (ej.: estudiantes

peruanos que vuelven a la

universidad para reparar

ventiladores para un hospital

local; estudiantes de las carreras

de salud en diferentes países del

mundo que realizan testeos,

confeccionan barbijos,

camisolines o producen alcohol

en gel y lo distribuyen en

respectivos territorios).

- AYSS híbrido o combinado: parte de las actividades se realizan virtualmente y parte de las actividades se realizan presencialmente (ej.: estudiantes que elaboraron y donaron máscaras de seguridad sanitarias en un espacio doméstico para luego llevarlas al hospital local).

- AYSS virtual: ocurre cuando las actividades se realizan

virtualmente sin que los "estudiantes salgan de sus casas" (por ejemplo: estudiantes universitarios españoles que se ofrecen para ayudar a preparar exámenes a otros estudiantes; estudiantes de Singapur que dan clases de "educación física virtual" para adultos mayores a los que no pueden acompañar presencialmente; tutorías o acompañamiento educativo virtual que realizan estudiantes universitarios argentinos).

En tiempos de profundas desigualdades se desafía la capacidad de crear y usar la información para acrecentar el aprendizaje, el servicio y la solidaridad en un mundo complejo. Si bien no contamos aún con estudios que releven y cuantifiquen estos tres ámbitos y modalidades de desarrollo del AYSS a nivel global, sí existen iniciativas tendientes a su registro y sistematización que abonan a una mayor interdependencia solidaria.

A su vez, la posibilidad de acceder a un escenario digital global permite una continuidad en las relaciones sociales, en las que personas, organizaciones y redes pueden interactuar y aprender juntas. La etnografía digital permitió así observar y dar cuenta de distintos comportamientos registrados, como los intercambios en los chats al conocer $y$ promover nuevas experiencias, el proceso de construcción del mapa, la identificación de buenas prácticas y su apertura, reflexión y difusión a través de los numerosos webinarios dictados por el equipo de CLAYSS y de las redes de aprendizaje-servicio a las que pertenece (Pink et al., 2016).

Es en tiempos de profundas crisis cuando se evidencia y pone a prueba la capacidad que poseen los Estados, las instituciones y las organizaciones para aprender y seguir innovando. Desde el AYSS estas capacidades no son sólo para sí sino en miras de un proceso de humanización del mundo que en medio del dolor haga aflorar lo mejor de sí. La teoría del análisis organizacional expresa que una gestión educativa estratégica se pone de manifiesto cuando las organizaciones disponen de repertorios de respuestas, que son a su vez una evidencia de su adaptación a los cambios. Por el contrario, una gestión de tipo "administración" mantiene funcionando lo dado, no contando con el habitus o la "gimnasia"

Tapia, M. R. y Peregalli, A. (2020). Aprender, servir y ser solidarios en tiempos de pandemias. RIDAS, Revista Iberoamericana de Aprendizaje Servicio, 10, 49-61. DOI10.1344/RIDAS2020.10.5 
para que esas adaptaciones sucedan y en la dirección u orientación propuesta (Instituto Internacional de Planeamiento de la Educación [IIPE]UNESCO, 2000).

El aprendizaje organizacional surge como resultado de un pensamiento estratégico que analiza las condiciones particulares en que se desarrolla la acción educativa. Supone apertura y facilitación de comunicación interna y externa; retroalimentación permanente con respecto a logros, carencias y demandas; evaluación de la mejora; acumulación de conocimiento y generación de innovaciones.

Las organizaciones abiertas al aprendizaje tendrán en cuenta la factibilidad y viabilidad de las acciones, entendiendo factibilidad como la posibilidad de realizarlas y viabilidad, como las condiciones del hacer: saber hacer (viabilidad técnico-pedagógica); querer hacer (viabilidad políticocultural); poder hacer (viabilidad organizativo administrativa)

(Aguerrondo, Lugo y Rossi, 2001).

Cuando las personas y las organizaciones miran para ver de nuevo y experimentan el mundo de forma más compleja y potente, comienzan a radicarse a nivel de la cultura institucional nuevas ideas que dan lugar a innovaciones (disrupciones profundas). En la medida en que esto suceda, se puede hablar de ciclo de aprendizaje; sin la constitución de bases para el desarrollo de organizaciones inteligentes, los cambios quedan en la superficie y no a nivel de creencias y hábitos (IIPE-UNESCO, 2000).

\section{Descripción de la experiencia}

El mapa "Experiencias de aprendizajeservicio solidario en tiempos de pandemias $^{1 "}$ (CLAYSS, 2020) ilustra un modo de ser organizacional y su contenido nutre el desarrollo de una organización que aprende, sirve y busca contribuir al crecimiento de una cultura fraterna y solidaria.

El mapa surge en pandemia a raíz de una actividad presencial que no pudo realizarse. El taller online, realizado el 26 de marzo de 2020, fue organizado por la Universidad Católica de EichstattIngolstadt junto a CLAYSS, en el marco del Programa UNISERVITATE ${ }^{2}$ (PorticusCLAYSS). El mapa fue inicialmente pensado para identificar el lugar de origen de los participantes del encuentro, ya que al pasar al formato virtual la convocatoria pudo extenderse a otros participantes que no podrían haber asistido presencialmente, y a modo de presentación, los participantes eran invitados a compartir las experiencias de aprendizaje-servicio que estaban desarrollando en el marco de la pandemia.

El recurso utilizado para la elaboración del mapa fue la plataforma digital Padlet, que ofrece la posibilidad de crear murales colaborativos, y de construir espacios donde se pueden presentar recursos multimedia como videos, audios, fotos o documentos. Entre las diferentes plantillas disponibles, brinda la opción de crear mapas, en los que los usuarios pueden

${ }^{1}$ http://www.clayss.org.ar/CLAYSS exp_pand 2 $020 . h$ tml

2 http://www.uniservitate.org 
señalar una ubicación y agregar la información que deseen compartir.

El mapa interactivo fue elegido en función de los usuarios y la propuesta de trabajo pensada para el taller virtual, a partir de las características:

- online;

- multilingüe;

- de alcance mundial;

- autoadministrable;

- para ser utilizado por diferentes actores que en forma sincrónica o asincrónica acceden remotamente.

Una vez desarrollada esta acción de capacitación, y notando lo motivante que resultó para los participantes saber qué acciones de AYSS estaban desarrollándose en otras instituciones a nivel internacional, desde CLAYSS se decidió sostener y promover mundialmente esta iniciativa de registro de experiencias.

A partir de la intervención de los participantes y la información recopilada, el mapa actualmente es consultado como:

- Recurso que releva experiencias de AYSS en diferentes niveles del sistema educativo en tiempos de pandemia.

- Recurso que releva experiencias de AYSS presenciales, híbridas o virtuales.

- Recurso acumulativo que puede relevar varias experiencias de una misma institución o región, y que permite visualizar redes colaborativas.

El mapa invita a recopilar experiencias de AYSS de instituciones educativas en distintos países del mundo que se encuentran desarrollando proyectos vinculados a la pandemia, y convoca a educadores a sumar los suyos y continuar aprendiendo unos de otros. La virtualidad permitió el involucramiento de diversos actores institucionales e interinstitucionales (redes) que fueron nutriendo su desarrollo y replicando en nuevas redes, aprendizajes y servicios solidarios las experiencias allí registradas.

Luego de su creación en el período inicial de la pandemia, el mapa se presentó como un recurso muy valorado en diferentes actividades organizadas por CLAYSS (por ejemplo: webinarios, conferencias, el ${ }^{\circ} 23$ Seminario Internacional de Aprendizaje Servicio Solidario - CLAYSS, Buenos Aires, agosto 2020) invitando a los participantes a sumar sus iniciativas al mapa. Del mismo modo, se difundió desde la página web de CLAYSS, desde sus redes sociales y las de muchos aliados y seguidores.

El mapa recopila hasta el momento más de 200 experiencias de cuatro continentes, entre las cuales, las más numerosas son las de América y Europa, visibilizando las regiones de más actividad con CLAYSS al menos hasta la fecha (Tabla 1).

Tapia, M. R. y Peregalli, A. (2020). Aprender, servir y ser solidarios en tiempos de pandemias. RIDAS, Revista Iberoamericana de Aprendizaje Servicio, 10, 49-61. DOI10.1344/RIDAS2020.10.5 
Tabla 1. CLAYSS. Cuantificación de experiencias de AYSS en pandemia

\begin{tabular}{|c|ccccc|}
\hline \multirow{2}{*}{$\begin{array}{c}\text { Cantidad de } \\
\text { experiencias } \\
\text { registradas }\end{array}$} & \multicolumn{4}{|c|}{$\begin{array}{c}\text { Cantidad de experiencias } \\
\text { registradas por continentes }\end{array}$} \\
\cline { 2 - 5 } & África & América & Asia & Europa & Oceanía \\
\hline 201 & 4 & 120 & 3 & 74 & ----- \\
\hline $100 \%$ & $2 \%$ & $60 \%$ & $1 \%$ & $37 \%$ & ---- \\
\hline
\end{tabular}

Fuente: elaboración propia

Al analizar el perfil de instituciones que registraron sus proyectos de AYSS, se destaca la preponderancia de las relativas a la Educación Superior (estatales y privadas). Esto podría explicarse sobre la base de que el mapa fue originalmente diseñado en el marco de actividades orientadas al desarrollo del AYSS en la Educación Superior, aunque abierto a utilizarse en lo educativo en general. Cabe señalar también que se referencian experiencias y proyectos impulsados desde variadas instituciones: de Educación Secundaria (con sus diferentes variedades, ej.: técnicas), de Educación Primaria e Inicial, de la Sociedad Civil, pertenecientes a redes de Organizaciones de la Sociedad Civil; impulsadas desde Ayuntamientos o Municipios y desde Institutos de Formación Docente.

La Tabla 2 detalla los países georreferenciados en el mapa (22 en total) y la cantidad de proyectos y experiencias de Aprendizaje y Servicio Solidario registrados en cada caso, presentados por orden alfabético.

Tabla 2. Proyectos y experiencias de AYSS en pandemia según países y cantidad de experiencias registradas

\begin{tabular}{|c|c|c|c|}
\hline \multicolumn{4}{|c|}{ País/Cantidad experiencias } \\
registradas \\
\hline Alemania & 5 & Italia & 4 \\
\hline Argentina & 76 & Kenia & 1 \\
\hline Azerbaiyán & 1 & México & 7 \\
\hline Brasil & 3 & Paraguay & 2 \\
\hline Chile & 11 & Perú & 6 \\
\hline Colombia & 4 & Portugal & 2 \\
\hline Costa Rica & 2 & Rumania & 2 \\
\hline Ecuador & 4 & Singapur & 1 \\
\hline España & 60 & Sudáfrica & 4 \\
\hline Estados Unidos & 1 & Ucrania & 1 \\
\hline India & 1 & Uruguay & 3 \\
\hline
\end{tabular}

Fuente: elaboración propia

En la siguiente Tabla (3) se sistematizan los proyectos y experiencias de AYSS registrados en el mapa, emergente del análisis de cada experiencia (su descripción). La sistematización implicó una tarea de síntesis y agrupamiento de las experiencias en función de su objetivo principal. Por dicha razón, a efectos de que el lector consulte el mapa y consigne proyectos y experiencias, se omiten los nombres y datos de identificación y se presentan algunas experiencias -a modo de ejemplo- para cada categoría identificada. 
Tabla 3. Sistematización de proyectos y experiencias de AYSS en pandemia

Sistematización de proyectos y experiencias de AYSS en pandemia

\section{Proyectos de AYSS para el acompañamiento y cuidado de personas hospitalizadas, personal sanitario, adultos mayores y personas con discapacidad a través de cartas, llamadas telefónicas, videollamadas y videos distribuidos online}

- $\quad$ Proyecto "Hay muchos abuelos sin medicación y con hambre".

- Publicación digital de herramientas para el diseño, implementación y evaluación de proyectos de aprendizaje servicio en artes en centros de día para personas mayores.

- Animación a la lectura en formato video (elaboración de materiales, cuentos, poesías, trucos de magia, raps...) para apoyar a pacientes y personal de hospitales y residencias de mayores.

- Proyecto para mandar mensajes de ánimo a personas mayores para animarlas y recordarles que aunque desde la distancia siguen presentes en la vida.

- $\quad$ "Cartas con corazón" y dibujos para pacientes hospitalizados.

- Proyecto "Mensaje de ánimo". Diversos centros educativos de la ciudad crean mensajes de ánimo para mayores y personas con discapacidad ante la situación de confinamiento.

- Proyecto de Institutos de Formación Docente al servicio de adultos mayores confinados y compartiendo todos los aprendizajes adquiridos (videos, contando cuentos, historias, realizando ejercicios, canto y baile en compañía virtual).

- Proyecto: Déjame acompañarte jugando.

- Proyecto "Cartas para todos los héroes y heroínas" que están luchando actualmente en nuestras calles contra el COVID-19.

- Programa: "'Soledad Cero"

- Proyecto: "Solidaridad sin cuarentena"

Proyectos de alfabetización digital y acompañamiento educativo virtual
- $\quad$ Proyecto de "ayuda socioeducativa para vencer la brecha digital y crear espacios analógicos" (apoyo escolar, buscar ordenadores, tablets, wifi y localizando a las personas que los necesiten y los puedan aprovechar; generando espacios y contenidos "analógicos" para los que, aunque dispusieran de medios digitales, no los sabrían utilizar.

- Construcción de comunidad virtual ("escuela en línea" para estudiantes coordinados por otros estudiantes).

- Videos con instrucciones para utilizar la plataforma digital de la escuela.

- Contribución entre escuelas hermanas.

- $\quad$ Facilitación online de colecciones de literatura infantil y juvenil, poniéndolo al servicio los sistemas educativos.

- Experiencia: 3D PMG. Proyecto para promover la alfabetización digital y científica en jóvenes de la localidad.

- $\quad$ Oportunidades de aprendizaje para la primera infancia.

- Acompañamiento educativo virtual.

- Proyecto (audio-cuentos) para el programa de Aulas Hospitalarias.

- $\quad$ Proyecto para reducir la brecha digital de las personas mayores mediante su empoderamiento, poniendo foco en su relación con la administración electrónica.

\section{Información, intercambio y transferencia de saberes}

- $\quad$ Creación de "cortos y spot" publicitarios para concientizar sobre la pandemia, en diferentes idiomas y formatos para compartir mensajes de apoyo y de prevención con estudiantes del resto del mundo.

- Servicio para concientizar sobre el buen uso del barbijo (proyecto curricular de Química).

- Talleres online con el objeto de ofrecer formación, reflexión y desarrollo de habilidades para la intervención social en el nuevo contexto.

- Seminarios web gratuitos para ancianos, cuidadores y organizaciones.

- Mapa de infraestructura social elaborado por estudiantes universitarios para veteranos de guerra de Ucrania.

Proyectos para la alimentación saludable

Tapia, M. R. y Peregalli, A. (2020). Aprender, servir y ser solidarios en tiempos de pandemias. RIDAS, Revista Iberoamericana de Aprendizaje Servicio, 10, 49-61. DOI10.1344/RIDAS2020.10.5 
- $\quad$ Revalorización del valor cultural de la transmisión de recetas tradicionales saludables (talleres).

- Entrega de canastas "saludables" y trabajo curricular con estos insumos desde todas las asignaturas de forma integrada, pesando, midiendo, elaborando recetas, realizando cálculos matemáticos y promoviendo una dieta equilibrada. Acompañamiento y reciprocidades entre familiascadenas de aprendizaje y servicio solidario.

- Proyecto: Alimentarse no tiene desperdicio.

\section{Proyectos para asistencia a familias en barrios vulnerables}

- Colectas para familias como parte de las actividades curriculares.

- $\quad$ Proyecto solidario "manos a la obra" (pastoral universitaria): acompañando y colaborando con varias actividades en el barrio.

\section{Proyectos para el desarrollo local: apoyo del comercio local, asesoría para emprendedores y orientación laboral}

- $\quad$ Proyecto Moraleja: una empresa de todos y todas. Apoyo al comercio local desde las aulas.

- "Apadrina un comercio vecino".

- Dispositivo para acompañar y asesorar a emprendedores.

- Servicio gratuito de asesoría y gestión comunicacional online para emprendedores de la región.

- Proyecto de Orientación Laboral COVID19 en el marco universitario.

\section{Proyectos de cuidado del medioambiente}

- Talleres ecológicos con enfoque en aprendizajeservicio.

- $\quad$ Arte reciclado con llantas, botellas y otros, para crear maceteros, asientos y jardines colgantes y entregarlos a la comunidad.

- Concientización sobre el agua potable en tiempos de pandemia.
- $\quad$ Proyecto interdisciplinario basado en la educación ambiental, sustentabilidad y educación emocional, a través del reciclado de diferentes materiales con los que realizan barbijos, juegos didácticos, macetas, los cuales son distribuidos entre las familias de la zona.

- Proyecto "Yo te ayudo con la basura": jóvenes voluntarios que llevan la basura del vecindario al punto de recogida con objetivo de minimizar riesgos de exposición al contagio.

\section{Proyectos de asistencia sanitaria}

- $\quad$ Estudiantes de bioquímica, kinesiología, enfermería o medicina atienden necesidades comunitarias con base a un proyecto que articula de manera constante con la comunidad.

- $\quad$ Línea telefónica directa para asistencia psicológica.

- Agilizar la obtención de turnos online para donar sangre.

- Voluntariado sanitario.

\section{Producción de insumos sanitarios}

- Impresión tridimensional de máscaras de higiene.

- Diseño de máscaras faciales y de bioseguridad en 3D y donación al personal de salud y al personal de seguridad de la comunidad.

- Realización de mascarillas para personas sordas.

- $\quad$ Producción de barbijos, camisolines y uniformes para personal médico que trabaja en la primera línea de detección del COVID 19.

- Realización y entrega de protectores faciales a familias que lideran las acciones solidarias.

- Elaboración de productos farmacéuticos para las personas que carecen de recursos económicos, incluida la producción y distribución de repelentes para fumigar los mosquitos y nebulizar sus hábitats en toda la localidad.

- $\quad$ Producción de productos de higiene y salud entre trabajadores y estudiantes de ciencia y tecnología y docentes de escuelas técnicas.

- Fabricación de portasueros metálicos por parte de Escuela Técnica.

- Video sobre limpieza y desinfección de equipos e instalaciones hecho por alumnos.

- $\quad$ Proyecto de reparación de ventiladores mecánicos para el hospital local.

Tapia, M. R. y Peregalli, A. (2020). Aprender, servir y ser solidarios en tiempos de pandemias. RIDAS, Revista Iberoamericana de Aprendizaje Servicio, 10, 49-61. DOI10.1344/RIDAS2020.10.5 


\section{Proyectos vinculados con los derechos, equidad y prevención de la violencia de género}

- $\quad$ Proyecto: "Conocé tus derechos durante el estado de alarma".

- Proyecto: "Descubriendo personas defensoras de los derechos humanos".

- Proyecto: "Clínicas jurídicas de universidades españolas".

- $\quad$ Proyectos para abordar situaciones de violencia de género a través de cápsulas radiofónicas en vinculación con el Ministerio de Educación. Se trabaja en línea con empleadas de empresas concretas sobre talleres para identificar la violencia de género.

- $\quad$ Programa de capacitación online a participantes del programa de Equidad de Género.

- Proyecto de igualdad de género (APS en tiempo de coronavirus).

\section{Promoción del AYSS / Programas institucionales de AYSS}

- $\quad$ Surgimiento de nuevas líneas de acción en Proyecto de AYSS ya existente (radio comunitaria se convierte en espacio de intercambio de aprendizajes referidos al turismo y cuidado de la salud frente a esta pandemia).

- $\quad$ Proyecto "¿Qué es y cómo se hace un proyecto de ApS?" (adolescentes que explican a otros adolescentes).

- Promover y articular acciones pertinentes de fraternidad y solidaridad, tanto para la etapa de cuarentena como de post-cuarentena.

- Investigación-acción-comunitaria desde Observatorios de Responsabilidad Social e Instituciones de Enseñanza Superior.

Fuente: elaboración propia

Como puede visualizarse en la Tabla 3, el contexto de la pandemia ha hecho emerger un sinfín de experiencias que muestran el vigor del AYSS en el mundo y sus variaciones y que utilizan para su desarrollo y difusión herramientas y medios digitales muy variados como: sitios web, blogs, Genial.ly, Padlet, y redes sociales como Instagram y Facebook principalmente.

\section{Los proyectos y experiencias son} variados en alcance, magnitud y grado de institucionalización. Algunos más cercanos a la definición de AYSS que compartimos previamente; otros, más cercanos a experiencias de voluntariado en tiempos de emergencia, indicando "corrimientos" propios de un momento de la humanidad que desafía la creatividad, la capacidad de innovación, la apertura al aprendizaje.

\section{Conclusiones}

La solidaridad busca las maneras de decir presente en tiempos de pandemia; las experiencias y proyectos de AYSS son resilientes evidencias de ello en un momento tan trágico de la humanidad. Aunque es muy incipiente hacer una evaluación a gran escala sobre el impacto de las acciones del AYSS y sus resultados, puede afirmarse que buscan vías y caminos para ejercer una acción política que contribuye a la construcción del espacio común.

El relevamiento realizado permite concluir acerca de la preponderancia de experiencias de AYSS híbrido o virtual por sobre las experiencias de tipo presencial. Estas últimas, sin embargo, muy relevantes en tiempos de profundas desigualdades, nos hablan de una ética del riesgo que se asume cuando el compromiso evidencia que importan los otros y que, en este mundo, y pese a todo, estamos llamados a construir encuentros. 
En tiempos de pandemias el AYSS se resignifica en la virtualidad, y con ello hay una reconfiguración de las organizaciones y de quienes las integran: individuos y colectivos sociales que experimentan nuevas subjetividades y nuevos modos de ser y estar en el mundo. En ese sentido este trabajo es la evidencia de un proceso más profundo, de un modo de ser organizacional e institucional, abierto al cambio y al aprendizaje, con capacidad adaptativa y resiliente, donde intervinieron diferentes actores que han enriquecido este proceso y se han visto enriquecidos. Esto habla de la construcción colaborativa de conocimiento, de visualizar el conocimiento distribuido y las redes que se consolidan con la experiencia solidaria.

\section{Referencias bibliográficas}

Aguerrondo, I., Lugo, M.T. y Rossi, M. (2001). La gestión de la escuela y el diseño de proyectos institucionales. Buenos Aires, Argentina: Universidad Nacional de Quilmes.

Centro Latinoamericano de Aprendizaje y Servicio Solidario [CLAYSS]. (2020). Algunas experiencias de aprendizajeservicio solidario frente a epidemias. Recuperado de: http://www.clayss.org.ar/CLAYSS exp pand 2020.html

CLAYSS Digital. (26 de agosto de 2020). Apertura y Conferencia inaugural Prof. Nieves Tapia [Archivo de Vídeo]. Youtube. https://www.youtube.com/watch?v=QX 5gP4xzgis
Instituto Internacional de Planeamiento de la Educación (IIPE-UNESCO). (2000). Gestión educativa estratégica. Módulo 2. Buenos Aires, Argentina: IIPE.

Ministerio de Educación. (2020). Informe preliminar: políticas educativas en el contexto internacional. Ciudad Autónoma de Buenos Aires, Argentina: Ministerio de Educación de la Nación. Recuperado de:

https://www.argentina.gob.ar/sites/def ault/files/politicas educativas en el co ntexto internacional.pdf

Pontificia Academia para la Vida. (30 de marzo de 2020). Pandemia y fraternidad universal. Recuperado de: http://www.vatican.va/roman curia/po ntifical academies/acdlife/documents/r $c$ pont-

acd life doc 20200330 pandemiafraternita-universale sp.html

Papa Francisco. (2020). La vida después de la pandemia. Ciudad del Vaticano: Libreria Editrice Vaticana.

Pink, S., Horst, H., Postill, J., Hjorth, L., Lewis, T. y Tacchi, J. (2016) Digital Ethnography: Principles and Practice. SAGE Publications Ltd.

Piro, I. (22 de julio de 2020). Covid-19. PAV: necesaria la ética del riesgo, solidaridad y cooperación internacional. Vatican News. Recuperado de: https://www.vaticannews.va/es/vatican o/news/2020-07/covid-19-pavnecesaria-etica-riesgo-cooperacioninternacional.html

Organización de las Naciones Unidas para la Educación, la Ciencia y la 
Cultura [UNESCO]. (2020), Impacto de la COVID-19 en la educación.

Seguimiento mundial de los cierres de escuelas causado por el Covid-19.

https://es.unesco.org/covid19/educatio

nresponse/

Tapia, M. N. (2000). La Solidaridad

como Pedagogía. Buenos Aires,

Argentina: Ciudad Nueva.

Waldner, L., McGorry, S. y Widener, M.

(2012). E-Service-Learning: The

Evolution of Service-Learning to Engage

a Growing Online Student Population.

Journal of Higher Education Outreach

and Engagement, 16(2), 123-149.

Recuperado de:

https://files.eric.ed.gov/fulltext/EJ9758

13.pdf 